\title{
Análise Praxeológica de livro didático de matemática referente ao estudo de números binários
}

\author{
Praxeological Analysis of a mathematics textbook \\ about the study of binary numbers
}

Herman do Lago Mendes

herman2000@zipmail.com.br

\begin{abstract}
Resumo
Objetiva analisar as organizações praxeológica matemática e praxeológica didática referente ao estudo de números binários em uma coleção de livro didático de matemática dos anos finais do ensino fundamental avaliado pelo Programa Nacional do Livro Didático (PNLD 2014). Utiliza a Teoria Antropológica do Didático (TAD) tanto para fundamentar teoricamente o trabalho como ferramenta de pesquisa. Mais especificamente, utiliza um dos conceitos da TAD - praxeologia - como ferramenta de pesquisa. Identifica abordagem de números binários por meio de dois domínios da educação básica de matemática: Grandezas e Medidas (medida de informação) e Números e Operações (sistema de numeração binário). Identifica na coleção: 3 tipos de tarefas, 3 técnicas, uma única tecnologia e nenhuma teoria. A abordagem dos números binários nos livros analisados (volumes 6 e 7) caracteriza-se como sendo praxeologia pontual e local.
\end{abstract}

Palavras-Chave: Praxeologia. Teoria Antropológica do Didático. Ensino Fundamental. Livro Didático de Matemática.

\begin{abstract}
It is the objective of this paper to analyze the mathematics praxeological and the didactic praxeological organizations regarding the study of binary numbers in a collection of mathematics textbook for the final years of the elementary school evaluated by the National Textbook Program (PNLD 2014). It uses the Anthropological Theory of Didactic (ATD) as a way to provide the theoretical support for the study. More specifically, it uses one of the TAD concepts - praxeology - as a research tool. It identifies the approach of binary numbers through two fields of mathematics basic education: Quantities and Measures (information measurement) and Numbers and Operations (binary numbering system). It identifies in the selection: 3 types of tasks, 3 techniques, a single technology and no theory. The approach about binary numbers in the analyzed books (volumes 6 and 7) is characterized as being punctual and local praxeology.
\end{abstract}

Keywords: praxeology, Anthropological Theory of Teaching, Elementary School, Mathematics Textbooks

\section{Introdução}

Verificamos a existência de abordagem de números binários em livros didáticos de matemática dos anos finais do Ensino Fundamental avaliados pelo Programa Nacional do Livro Didático (PNLD 2014). Objetivamos analisar as organizações praxeológica matemática e praxeológica didática referente ao estudo de números binários em livro didático de matemática dos anos finais do ensino fundamental avaliado pelo PNLD 2014. Utilizamos a Teoria Antropológica do Didático (TAD) tanto para fundamentar teoricamente este trabalho 
como ferramenta de pesquisa. Mais especificamente, utilizamos um dos conceitos da TAD praxeologia - como ferramenta de pesquisa.

A TAD defende a existência de princípios inter-relacionados de elementos práticos e teóricos de qualquer que seja a ação humana. E a partir disso, as atividades matemáticas - que por sinal são consideradas como um exemplo de ações humanas - são elaboradas e executadas para algum fim, que por sua vez são advindas de pelo menos um questionamento, que é apoiado por elementos lógicos e teóricos embutidos. A partir disso, a TAD, mais especificamente, a praxeologia ou a organização praxeológica - um dos elementos conceituais da TAD - defende a existência de justificação e explicação de qualquer que seja a ação, o fazer humano.

Praxeologia deriva de duas palavras gregas, práxis e logos; são princípios inter-relacionados de elementos práticos e teóricos na ação humana em realizar algum fim: práxis significa a parte prática, o saber-fazer, enquanto o logos significa a parte inteligível, racional e lógico desse saber-fazer.

\section{Praxeologia}

A noção de tarefa é a raiz da noção de praxeologia. Uma tarefa $t$ pertence a um tipo de tarefa $T(t \in T)$ ou seja, na maioria dos casos uma tarefa é um tipo de "tarefa pai" e é expresso por um verbo: caminhar pela praça, escovar os dentes, somar dois números inteiros, etc. Yves Chevallard destaca três características imediatamente notadas para o conceito de tarefa: primeiro, o conceito de tarefa ou tipos de tarefas é amplo, um simples coçar os olhos, sorrir para alguém já é considerado uma tarefa. Suponha que uma médica solicita ao seu paciente, que tem problemas de locomoção motora, mexa os dedos dos pés. Esta tarefa pode servir (ou não) de procedimento para a médica realizar alguma atividade diagnóstica. O conceito de tarefa, ou melhor de tipo de tarefa, requer um propósito relativamente específico (CHEVALLARD, 1998). Segundo, um tipo de trabalho apenas existe na forma de diferentes tipos de tarefas e o seu conteúdo é rigidamente especificado. Exemplo: a tarefa - calcular algo - em determinado nível de escolaridade pode ser enriquecida, no mesmo nível de ensino ou em outro nível maior, por novos tipos de tarefas: construir, mostrar, ordenar, etc; Terceiro, tarefas e tipos de tarefas são produções, "artefatos", "obras" humanas, construções intencionadas.

Partindo daqueles três pontos referentes à tarefa, esta estaria relacionada ao "fazer coisas". Yves Chevallard a considera como um elemento de organização praxeológica estática, uma vez que a dinâmica necessária para a sua execução é, a princípio, não revelada. Nessa 
dinâmica podem existir maneiras diferentes de realizar, de executar tais tarefas, que segundo esse mesmo pesquisador, o chama de técnica. Esta é considerada como a "arte", um saber comum, surgida do grego techne. Assim, uma praxeologia sobre um tipo de tarefa $T$ contém, em princípio, uma técnica $\tau$ em T. Estes dois elementos da organização praxeológico é definido por Chevallard (1998) como sendo um "bloco"

$[\mathrm{T}, \tau$,] chamado bloco prático-técnico. Que genericamente é constituído como saber comum.

Para que a técnica $\tau$ possa exercer, executar, realizar determinados tipos de tarefas devem ser considerados três pontos (CHEVALLARD, 1998): primeiro, uma técnica é o "como fazer" de determinado tipo de tarefa. Sucede apenas uma parte $\mathrm{P}(\tau)$ do tipo de tarefa que se refere ao escopo da técnica. Exemplo: Amarildo faz barba utilizando uma navalha. Primeiro ele molha o rosto com água e sabão e em seguida passa a navalha de cima para baixo raspando os pelos do rosto. Bruno faz barba utilizando uma máquina própria. Primeiro ele apara os pelos do rosto com uma tesoura e o restante, de forma circular, utiliza essa máquina para raspar os pelos do rosto. Carlos faz barba utilizando um barbeador. Primeiro ele passa um pano molhado e um pouco quente no rosto e em seguida passa as lâminas do barbeador, do sentido de baixo para cima para raspar os pelos do rosto. Amarildo, Bruno e Carlos utilizam técnicas diferentes, porém realizam o mesmo tipo de tarefa: fazer barba; Segundo, a técnica sendo ela generalizada é identificada como "saber comum"; Terceiro, cada instituição pode trabalhar técnicas diferentes ou iguais a outras instituições, uma instituição pode ignorar ou não (re)conhecer uma técnica utilizada por outra instituição.

Partindo daqueles três pontos referentes à técnica, esta estaria relacionada ao "como fazer coisas", maneira de realizar, de executar as coisas, as tarefas. Por outro lado, uma pessoa poderia perguntar por que Carlos passa uma toalha molhada e um pouco quente no rosto antes de fazer a barba. Ele poderia responder: para que os poros do meu rosto fiquem mais abertos, facilitando a raspagem dos pelos e não os deixando encravar. Esta justificativa e explicação da técnica utilizada por Carlos é considerado por Chevallard como outro elemento de organização praxeológica, chamado de tecnologia. O termo tecnologia $\theta$ é um discurso racional (logos) sobre a técnica $\tau$. É a explicação e justificação, de maneira racional, da técnica. De tal forma que a técnica garanta executar, realizar a tarefa do tipo $\mathrm{T}$ de maneira eficiente e lógica. Em muitos casos, elementos tecnológicos são integrados na arte.

Chevallard (1998) destaca três pontos referentes ao entendimento de tecnologia: primeiro, a função da tecnologia é justificar a técnica; Segundo, a função da tecnologia é explicar, de maneira inteligível determinada técnica. Assim, a primeira função da tecnologia seria REVEMAT. Florianópolis (SC), v.10, n. 1, p. 199-219, 2015. 
necessária para garantir a eficiência da técnica e a segunda função seria necessária para poder explicar o seu por quê; Terceiro, existem tecnologias potenciais, técnicas pendentes que são poucas técnicas.

Partindo daqueles três pontos referentes à tecnologia, esta estaria relacionada ao "por que fazer coisas de determinada maneira". A tecnologia é o como fazer as coisas de maneira racional. Ela é intrínseca, entrelaçada com a técnica. É a justificação e a explicação de funções das técnicas utilizadas para realizar determinadas tarefas ou tipos de tarefas. Por outro lado uma pessoa ainda poderia perguntar por que os poros da pele do rosto abrem ao manter contato com a água morna. Uma explicação ainda racional dessa justificação poderia ser dada pelo processo de dilatação térmica. Assim, o discurso tecnológico contém afirmações que pode conter porquês. E a partir destes porquês, ao maior nível de explicação dela, surge mais um elemento da organização praxeológica chamada por Chevallard (1998) de teoria $\Theta$. Ou seja, a teoria $\Theta$ é o porquê das tecnologias utilizadas nas explicações e justificações das técnicas. "A teoria refere-se a um conjunto mais abstrato de conceitos e argumentos dispostos em um discurso geral que justifica a tecnologia em si” (MARTENSEN, 2011, p.218). A teoria é entrelaçada a tecnologia e ambas são definidas por Yves Chevallard como sendo um bloco tecnológico-teórico ${ }^{[\theta, \Theta]}$.

A praxeologia ou organização praxeológica é constituída por um bloco prático-técnico $[\mathrm{T}, \tau]$ e por um bloco tecnológico-teórico $[\theta, \Theta$ ]. Segundo Chevallard (1998), o primeiro bloco é normalmente identificado como conhecimento enquanto que o segundo bloco é normalmente identificado como saber comum.

"O desenvolvimento de técnicas está no coração de atividade matemática" (CHEVALLARD, 1998, p.23 tradução nossa). O estudo e a resolução de um determinado tipo de problema (tarefas) podem estimular a criação de pelo menos uma técnica. Assim, o estudo de um determinado problema não é um fim a si mesmo, mas aparece como meio de construção de uma técnica capaz de resolvê-la ou não. Independente de determinada técnica irá resolver ou não a tarefa, existe a intenção em buscar resolver o problema e com isso desenvolve uma dialética fundamental de criação e de aprofundamento, estudo de problemas técnicos relacionados com o mesmo tipo de técnica que a partir daí, a própria técnica poderá ser um procedimento para resolver o problema deste tipo.

Segundo Chevallard (1998), geralmente um tipo de tarefa é definido em relação a um ambiente tecnológico-teórico previamente desenvolvido ou estimulado para criar um 
ambiente dialético com o surgimento das justificações e explicações eficientes de determinada técnica (tecnologia).

A partir daquele ambiente dialético existente de estímulo ao estudo de criações de técnicas mais eficientes do que as já existentes para executar um tipo de tarefa parte, segundo Chevallard (1998), do processo de institucionalização. A partir daqui surge à criação de regras, de critérios, de normas, de interesses, de objetivos que especificam o que deve ou não ser organizado (matematicamente). Etapa que irá definir o que são prioridades, o que são levados, matematicamente, em segundo, terceiro... plano.

Segundo Martensen (2011) o modelo praxeológico foi recentemente utilizado para a análise e projeto de intervenção de ensino em contextos formais de educação científica onde uma de suas contribuições tem sido a identificação e remediação de dissociação entre teoria e prática do corpus de conhecimentos ensinados.

Chevallard (1998) define quatro categorias de organizações praxeológicas: praxeologia pontual, praxeologia local, praxeologia regional e praxeologia global. A princípio, um tipo de tarefa $\mathrm{T}$ é formado por uma técnica $\tau$, uma tecnologia $\theta$ e uma teoria $\Theta$, todos estes denotados por praxeologia pontual $[\mathrm{T}, \tau, \theta, \Theta]$. A praxeologia pontual $[\mathrm{T}, \tau, \theta, \Theta]$ é assim categorizada quando apenas leva em consideração uma única tarefa T. A praxeologia local $[\mathrm{Ti}, \quad \tau \mathrm{i}, \theta, \Theta]$ é assim categorizada quando leva em consideração uma determinada tecnologia $\theta$. Geralmente uma determinada instituição I tem uma teoria $\Theta$, tem várias tecnologias $\theta_{\mathrm{j}}$ que cada qual por sua vez justifica e torna-se inteligível. Várias técnicas $\tau_{\mathrm{ij}}$ correspondem ao maior número de tipos de tarefas $\mathrm{T}_{\mathrm{ij}}$. Esse tipo de desenvolvimento em torno de uma única teoria é chamada de praxeologia regional [Tij, $\tau$ ij, $\theta_{\mathrm{j}}, \Theta_{\text {] }}$. Quando a organização praxeológica é desenvolvida em uma determinada instituição I pela agregação de

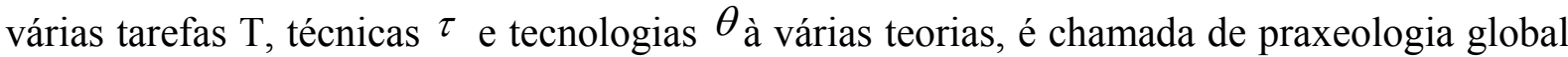
[Tijk, $\tau \mathrm{ijk}, \theta_{\mathrm{jk}}, \Theta_{\mathrm{k}}$. Ou seja, a praxeologia global é a agregação de várias organizações praxeológicas regionais atuantes às várias teorias $\Theta$.

Segundo Chevallard (1998), em muitos casos o tipo de tarefa T bloqueia o bloco tecnológicoteórico $[\theta, \Theta]$. Ou seja, há, em muitas instituições I, uma maior visibilidade no bloco práticotécnico ${ }^{[\mathrm{T}, \tau]}$; Da passagem da praxeologia pontual à praxeologia local a tecnologia é enfatizada. De maneira semelhante, da passagem da praxeologia regional à praxeologia global a teoria é trazida à tona. 
Segundo Chevallard (1998), mesmo a organização praxeológica pontual geralmente não é totalmente definida estruturalmente como: a técnica como sendo a forma de fazer, de realizar determinada tarefa, a tecnologia como sendo a justificativa e explicação dessa técnica utilizada para fazer essa tarefa e que a teoria como sendo o porquê desta justificativa para funcionar perfeitamente. Ou seja, esses elementos de organização praxeológica podem não ser tão bem definidos, perceptíveis na prática. "O tipo de tarefa em torno do qual foi constituída uma praxeologia pode não estar bem identificado, a técnica associada pode se revelar impraticável. A tecnologia pode, por vezes, ser reduzida a uma pura declaração de princípios e a teoria, pode ser perfeitamente enigmática" (ibidem, p.6 tradução nossa). Ou seja, a teoria pode não estar explicitada, assim como a tecnologia, a técnica...

Vale destacar que a organização praxeológica matemática parte de um tratamento didático para/com a seleção, organização, planejamento, método, entre outras preocupações didáticas que, de maneira imbricada, permeia, também, a existência de organização praxeológica didática. No momento que determinada instituição ou pessoa (o docente, por exemplo) permite a construção, ou melhor, a transposição de determinado objeto do saber (matemático) em objeto do saber a ser ensinado, articula-se (re)construções de tarefas, de técnicas, de tecnologias e de teorias, assim como em qualquer outra organização praxeológica. Portanto, segundo Chevallard (1998), a organização praxeológica didática ou praxeologia didática são respostas que objetivam satisfazer, realizar as seguintes perguntas: como estudar a questão q = $\tau_{\mathrm{T}}$ ?; Como estudar o objeto do saber $\mathrm{O}$ ?

A TAD define o trabalho do objeto (matemático) $\mathrm{O}$ como sendo uma combinação de elementos praxeológicos $[\mathrm{T}, \tau, \theta, \Theta$ ]. Didaticamente, o estudo de $\mathrm{O}$ consiste em providenciar alguma análise praxeológica de $\mathrm{O}$. O sujeito $\mathbf{x}$ (aluno) em fazer algo ajudado por $\mathbf{y}$ (professora) de maneira intencionada, compromete-se ao trabalho didático o que y requer para tal analisar a estrutura praxeológica de $\mathrm{O}$, assim como o seu estudo. Além disso, qualquer análise didática implica alguma (re)formulação de organização praxeológica de $\mathrm{O}$. (CHEVALLARD, 2013).

Chevallard (1998) sugere 6 momentos didáticos ou momentos de estudos, não necessariamente na mesma sequência, para assim possibilitar a análise de organização praxeológica didática:

Momento de (re)encontro com a praxeologia estudada (no nosso caso a praxeologia matemática) de tal maneira que satisfaça o objeto $\mathrm{O}$ com algum tipo de tarefa $\mathrm{T}_{\mathrm{i}}$; 
Momento de exploração do tipo de tarefa $T_{i}$ e a sua articulação com alguma técnica $\tau_{i}$ : constituição de determinadas técnicas de resolução;

Momento de constituição do bloco tecnológico-teórico: busca manter uma ligação com os demais momentos. Mantendo assim, justificativas e explicações para tais técnicas;

Momento de institucionalização: elaboração de objetivos, decisões, seleções, condições, regras a serem tomadas em qualquer momento didático;

Momento de trabalhar a técnica: tanto permeia a exercitação como também a performance de determinada técnica;

Momento de avaliação: articula o momento de institucionalização, refletindo, questionando, avaliando as relações pessoais e verificando eficiências em cada momento e relações entre os momentos didáticos.

No caso da praxeologia $\mathrm{O}=[\mathrm{T}, \tau, \theta, \Theta]$ existem momentos em algumas situações didáticas em que o sujeito $\mathbf{x}$ encontra-se um tipo de tarefa $T_{i}$ em um primeiro momento e, em seguida, depara-se, ou melhor, necessita-se de pelo menos uma técnica, atrelada a tarefa, para realizála, executá-la. O modelo praxeológico ${ }^{[\mathrm{T}, \tau, \theta, \Theta]}$ é quem dita o modelo de momentos didáticos.

"A didática é a ciência do ensino e, mais amplamente, das condições e das restrições aplicáveis à divulgação de entidades praxeológicas com as instâncias da sociedade" (CHEVALLARD, 2011, p.1 tradução nossa).

A necessidade em resolver, responder, realizar tarefas no campo da Matemática, de maneira imbricada, e até muitas vezes imperceptível, parte da organização praxeológica didática. Esta, por sua vez, é de natureza didática que nasce da necessidade de estudar como estudar determinada tarefa, como estudar determinado objeto do saber matemático. Sendo assim, a praxeologia didática possibilita (re)construir transposições de determinada praxeologia matemática.

\section{Procedimentos Metodológicos}

Verificamos a existência de números binários em livros didáticos de matemática dos anos finais do ensino fundamental avaliado pelo PNLD 2014 por meio de dois domínios ${ }^{1}$ :

${ }^{1}$ O PNLD define atualmente cinco blocos de conteúdos da Matemática básica: Números e Operações; Álgebra; Geometria; Grandezas e Medidas e Estatística e Probabilidade. 
Grandezas e Medidas (medida de informação) e Números e Operações (sistema de numeração binário). Escolhemos uma única coleção ${ }^{2}$. A partir daí, realizamos uma análise praxeológica (matemática e didática). Escolhemos esse livro porque ele aborda o sistema de numeração binário e unidades de medida de informação no livro do aluno e/ou no Manual do Professor.

Realizamos o seguinte procedimento de análise:

- Selecionamos os capítulos e/ou seções destinados aos números binários;

- Lemos os capítulos e/ou seções selecionados;

- Analisamos a organização praxeológica matemática referente ao estudo de números binários, composta por: identificação de tipos de tarefas, técnicas, tecnologias e teorias; caracterização do livro didático por meio de organizações praxeológicas: pontuais ou locais ou regionais ou globais;

- Analisamos a organização praxeológica didática referente ao estudo de números binários, recorrendo aos 6 momentos didáticos sugeridos por Chevallard (1998): momento de encontro com a praxeologia estudada, momento de exploração do tipo de tarefa e a sua articulação com alguma técnica, momento de constituição do bloco tecnológico-teórico, momento de institucionalização, momento de trabalhar a técnica e momento de avaliação;

Apesar do livro didático de matemática analisado trabalhar o sistema decimal, kilobyte, megabyte, gigabyte,... Iremos seguir as nomeações e simbologias literais recomendadas pelo Sistema Internacional de Unidades (SI, 2012) e pela Comissão Eletrotécnica Internacional (CIE, 2005): kibibyte (KiB), mébibyte (MiB), gibibyte (GiB), etc. Quando necessário, para: resolver tarefas ausentes de técnicas nos livros didáticos e para realizar explicações, argumentações necessárias às nossas análises.

\section{Análise praxeológica matemática frente ao estudo de números binários}

Identificamos dois tipos de tarefas no volume $7, \mathrm{~T}_{71}$ e $\mathrm{T}_{72}$, e, uma apenas, no volume $6, \mathrm{~T}_{61}$. $\mathrm{O}$ tipo de tarefa $\mathrm{T}_{71}$ configurou-se em transformar unidades de medida de informação. Presente na questão 81 (itens a e b) da página 113.

\footnotetext{
${ }^{2}$ A coleção analisada é a $1^{\text {a }}$ edição (ano 2012) da Editora Ática dos $6^{\circ}$ aos $9^{\circ}$ anos do Ensino Fundamental do autor Luiz Roberto Dante.
} 
Exemplo de tarefa do tipo de tarefa $\mathrm{T}_{71}$ : "Transforme 1,9 ZB em gigabytes (GB)" (DANTE, 2012b, p.113).

$\mathrm{O}$ tipo de tarefa $\mathrm{T}_{72}$ configurou-se em determinar a quantidade de artefatos tecnológicos (digitais) necessários para armazenar determinada medida de informação. Presente na questão 81 c da página 113.

Exemplo de tarefa do tipo de tarefa $\mathrm{T}_{72}$ : "Quantos pendrives de $8 \mathrm{~GB}$ são necessários para armazenar 92116 terabytes?" (DANTE, 2012b, p.113).

$\mathrm{O}$ tipo de tarefa $\mathrm{T}_{61}$ configurou-se em determinar a quantidade de informação existente em um determinado artefato tecnológico. Presente na questão 15 da página 231.

Exemplo de tarefa do tipo de tarefa T1: “[...] Escreva estas medidas, usando o byte como unidade e a potenciação: a) medida da capacidade de armazenamento do disco rígido" (DANTE, 2012a, p.231, ex: 15).

Todas as tarefas do volumes 6 e 7 recorrem a uma subtarefa (conjunta): converter unidades de medida de informação mantendo o número como uma potência de base 2 .

Identificamos três técnicas: uma (técnica $\tau_{1}$ ) para realizar conversões entre unidades de medidas de informação e duas técnicas (técnicas $\tau_{2}$ e $\tau_{3}$ ) para converter um número representado no sistema decimal em um número representado no sistema binário. Baseado nos enunciados das questões presentes no volumes 6 e 7 e na técnica de conversões entre unidades de medida de mesma grandeza (comprimento, capacidade, área, entre outras) presente no volume 6 (Figura 1), definimos a técnica $\tau_{1}$ como sendo a conversão entre unidades de medida de informação por meio do deslocamento de vírgula (produto ou divisão por 1024, ao invés de 1000 - base 10 - sistema de unidade métrico decimal) imbricada pela representação dos números como potencias de base 2 (recorrendo a propriedade da potenciação). A técnica $\tau_{2}$ presente no Manual do Professor, volume 6, página 67, configurou-se em transformar um número representado no sistema decimal em um número representado no sistema binário por

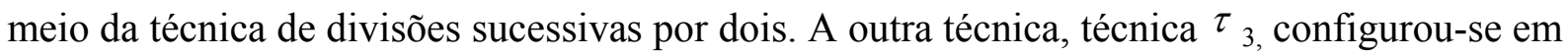
transformar um número representado no sistema decimal em um número representado no sistema binário por meio de sua escrita como uma expressão polinomial numérica de potência de base dois (Figura 5Figura 1). Vale destacar que as técnicas $\tau_{2}$ e $\tau_{3}$ foram ilustradas sem a solicitação de tarefas; não existem justificativas para as suas veracidades. 
Figura 1: Exemplo de técnica adotada para converter unidades de medida de mesma grandeza: deslocamento de vírgula - volume 6

\section{Transformações envolvendo as unidades de medida de capacidade}

As transformações das unidades de medida de capacidade são feitas de modo semelhante ao que já fizemos com as unidades de medida de comprimento e de massa. Observe a seguir as unidades de capacidade e como elas se relacionam.

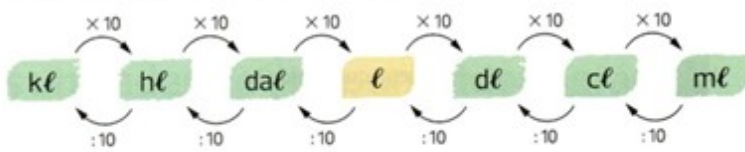

Exemplos:

a) Transformar 350 mililitros em litros.

Como o litro $(\ell)$ está três posições à esquerda do mililitro $(\mathrm{m} \ell)$, devemos dividir por 1000 .

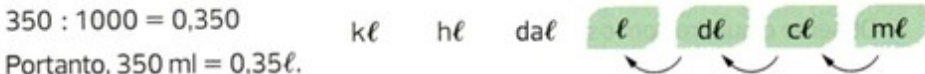

b) Transformar 3,875 hectolitros em centilitros.

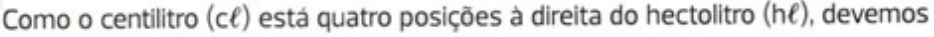
multiplicar por 10000 .

$3,875 \times 10000=38750 \mathrm{k} \ell \mathrm{h} \ell \mathrm{da} \ell \mathrm{\ell} \boldsymbol{\mathrm { C } \ell} \mathrm{m}$

Portanto, $3,875 \mathrm{~h} \ell=38750 \mathrm{c \ell}$.

Fonte: (DANTE, 2012a, p.241)

Como não identificamos resolução de tarefas envolvendo medidas de informação, propomos, a seguir, a resolução de todas as tarefas do volumes 6 e 7 baseados na técnica ${ }^{\tau_{1}}$ e adotando as nomenclaturas e simbologias do SI (2014) e do CIE (2005):

Os passos (técnica $\tau_{1}$ ) para resolver a tarefa da questão $81 \mathrm{~b}$ da página 113 (tipo de tarefa $\mathrm{T}_{71}$ ) são:

Primeiro: converter $1 \mathrm{ZiB}$ em gibibyte:

\section{$\times 1024 \times 1024 \times 1024 \times 1024$}

$1 \mathrm{GiB} \quad 1 \mathrm{TiB} \quad 1 \mathrm{PiB} \quad 1 \mathrm{EiB} 1 \mathrm{ZiB}$. Ou seja, $1 \mathrm{ZiB}=1024 \times 1024 \times 1024 \times 1024 \times 1 \mathrm{GiB}$

Segundo: escrever os produtos como potências de base 2:

$2^{10} \times 2^{10} \times 2^{10} \times 2^{10} \mathrm{GiB}=2^{40} \mathrm{GiB}$

Terceiro: multiplique por 1,9: $1,9 \times 2^{40} \mathrm{~GB}$ 
Os passos (técnica $\tau_{1}$ ) para resolver a tarefa da questão 81c da página 113 (tipo de tarefa $\mathrm{T}_{72}$ ) são:

Primeiro: converter 1 tébibyte em gibibyte:

$\mathrm{x} 1024$

$1 \mathrm{GiB} \quad 1 \mathrm{TiB}$. Ou seja, $1 \mathrm{TiB}=1024 \times 1 \mathrm{GiB}=1024 \mathrm{GiB}$

Segundo: multiplicar 92116 por $1024 \mathrm{GiB}$ : $94326784 \mathrm{GiB}$

Terceiro: dividir esse produto por 8: 11790848

Os passos (técnica $\tau_{1}$ ) para resolver a tarefa da questão 15a da página 231 (tipo de tarefa $\mathrm{T}_{61}$ ) são:

Primeiro: converter todas as medidas de informação em byte:

x1024 x 1024 x $1024 \approx$

1B $1 \mathrm{KiB} \quad 1 \mathrm{MiB} \quad 1 \mathrm{GiB}$. Ou seja, $1 \mathrm{GiB}=1024 \times 1024 \times 1024 \times 1 \mathrm{~B}$

$750 \mathrm{GiB}=750 \times 1024 \times 1024 \times 1024 \mathrm{~B}$.

Segundo: transformar os 1024 em potências de 2: $750 \times 2^{10} \times 2^{10} \times 2^{10} \mathrm{~B}$

Terceiro: realizar o produto conservando a base $2: 750 \times 2^{30} \mathrm{~B}$

Os passos (técnica $\tau_{1}$ ) para resolver a tarefa da questão $15 \mathrm{~b}$ da página 113 (tipo de tarefa T2) são:

Primeiro: converter todas as medidas de informação em byte:

$$
\times 1024 \times 1024
$$

1B $1 \mathrm{KiB} 1 \mathrm{MiB}$. Assim, $1 \mathrm{MiB}=1024 \times 1024 \times 1 \mathrm{~B}, \log 256 \mathrm{MiB}=256 \times 1024 \times 1024 \mathrm{~B}$

Segundo: transformar os 1024 em potências de $2: 2^{8} \times 2^{10} \times 2^{10} \mathrm{~B}$

Terceiro: realizar o produto de potências de 2 do segundo passo: $2^{28} \mathrm{~B}$ 
Definimos a tecnologia $\theta_{1}$, presente no volume 7 , como sendo a justificativa da técnica $\mathrm{T}_{1}$ que está enquadrada como tema: "armazenamento de dados na informática" (DANTE, 2012b, p.113, ex: 81):

\begin{abstract}
O bit é a menor unidade de informação que armazenamos na forma de dados digitais, seja em computadores, tablets, pendrives ou na própria internet [...] Unidades como bits e bytes estão organizadas sobre a base 2, pois um bit pode assumir apenas dois valores, 0 ou 1 (sistema binário). Assim, um byte, entendido como unidade, corresponde a $2^{0}=1$. Um quilobyte corresponde a $2^{0} \cdot 2^{10}=2^{10}$ bytes; um megabyte corresponde a $2^{10} \cdot 2^{10}=2^{20}$ quilobytes; um gigabyte equivale a $2^{20} \cdot 2^{10}$ $=2^{30}$ megabytes [...] (DANTE, 2012b, p.113, ex: 81).
\end{abstract}

Além disso, é definido, no volume 7, as seguintes unidades de medidas de armazenamento e de processamento de dados:

$\mathrm{B}=$ byte (uma unidade de informação); KB = kilobyte (1000 bytes);

$\mathrm{MB}=$ megabyte (1000 KB ou 1000000 bytes);

$\mathrm{GB}=$ gigabyte (1000 MB ou 1000000000 bytes $)$;

$\mathrm{TB}=$ terabyte $(1000 \mathrm{~GB}$ ou 1000000000000 bytes $) ; \mathrm{E}$

$\mathrm{MHz}=$ megahertz $(1000000 \mathrm{~Hz}$ ou 1000000 de ciclos por segundo);

$\mathrm{GHz}=$ gigahertz (1000 MHz ou $1000000000 \mathrm{~Hz}$ ou 1000000000 de ciclos por segundo)

A teoria referente às unidades de medidas de armazenamento e de processamento de dados é ausente no volume 6 e no volume 7 .

\title{
Análise praxeológica didática frente ao estudo de números binários
}

Momento de encontro com a praxeologia estudada: o livro do $6^{\circ}$ ano, capítulo 8 , sob o título: "Explorando a ideia de medida" aborda as grandezas de comprimento, superfície, massa, volume, capacidade e sob o título: "Outras grandezas" aborda, na página 229, grandezas usadas na informática: informação e velocidade de processamento de dados (Vide Figura 2). 
Figura 2: Introdução ao conteúdo de medidas de informação no volume 6

\section{Outras grandezas}

Você com certeza já esteve em situações que envolveram medidas de outros tipos de grandeza, como temperatura, tempo, velocidade, etc.

Vejamos mais duas, importantes no dia a dia da sociedade moderna.

\section{Grandezas usadas na informática}

Observe o significado destas unidades de medida usadas na informática:

Quantidade de informação
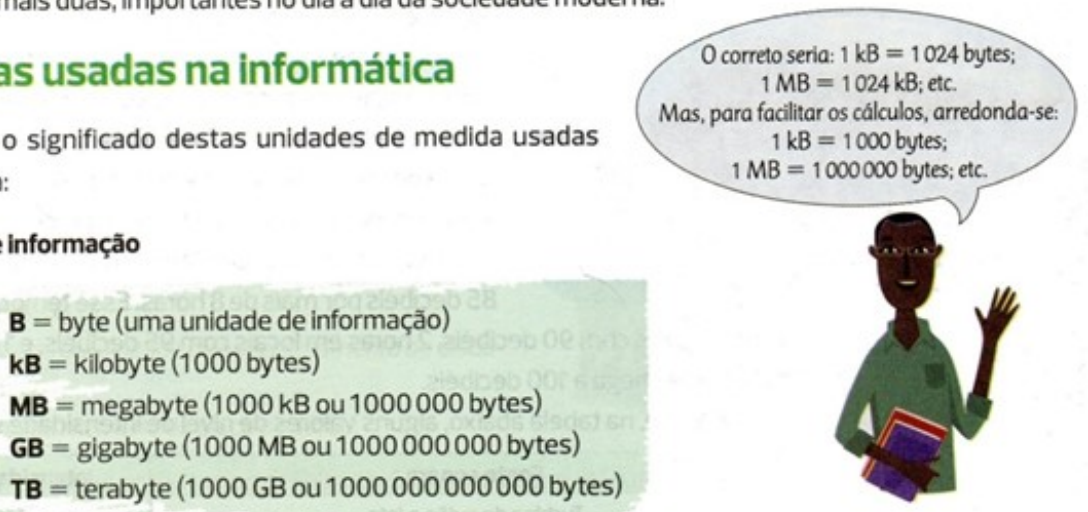

Velocidade de processamento de dados

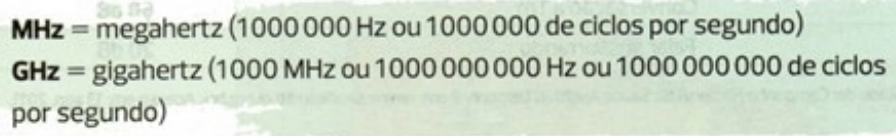

Usando potências de 10, podemos escrever:

- $800 \mathrm{MHz}=800000000 \mathrm{~Hz}=8 \cdot 10^{8} \mathrm{~Hz}$ (ciclos por segundo);

- $128 \mathrm{MB}=128000000 \mathrm{~B}=128 \cdot 10^{6} \mathrm{~B}$ (bytes).

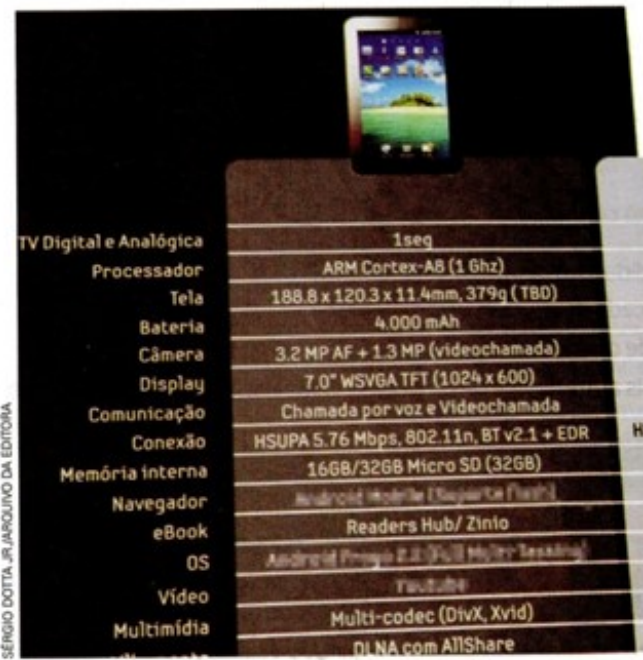

Cartaz publicitario de toblets, no qual aparecem várias unidades de medida relacionadas ainformattica.

Fonte: (DANTE, 2012a, p.229)

O livro do $7^{\circ}$ ano aborda medidas de informação no capítulo 3.8 - Números racionais- com o subtítulo "Outros contextos". A abordagem de medidas de informação no volume 7 é apresentada por meio da questão 81 (Figura 3). Enquanto que no volume 6 essa abordagem, e de medidas de velocidade de processamento de dados, são apresentadas no próprio tópico (tópico 3: "A ideia de medida e as várias grandezas") do capítulo 8: "Explorando a ideia de 
medida" (Figura 2). Os livros do $8^{\circ}$ e $9^{\circ}$ anos não abordam números binários por meio de sistema de numeração e nem por meio de unidades de medida de informação.

Figura 3: Questão 81 - Outros contextos

81. Armazenamento de dados na informática Como você estudou no ano anterior, hà diferentes grandezas e medidas relacionadas à informática, como, por exemplo, as que envolvem armazenamento de dados. Hoje em dia, é surpreendente a quantidade de informação que armazenamos na forma de dados digitais, seja em computadores, tablets, pen drives ou na propria internet.

$O$ bit é a menor unidade de informação que pode ser armazenada ou transmitida. Um conjunto de 8 bits corresponde a 1 byte. No ano anterior, você viu que as unidades do Sistema Métrico Decimal de Medidas (por exemplo, metro, litro, grama, etc.) es-

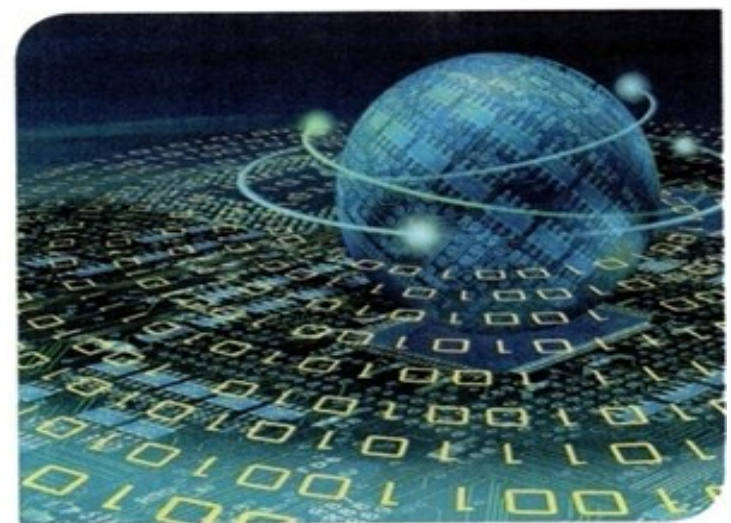
tão organizadas sobre a base 10. Ou seja, a partir de uma unidade-padrão ou unidade fundamental, as demais unidades de medida são obtidas multiplicando-se ou dividindo-se por potências de base 10 .

Jà unidades como bits e bytes estão organizadas sobre a base 2, pois um bit pode assumir apenas dois valores, 0 ou 1 (sistema binário). Assim, um byte, entendido como unidade, corresponde a $2^{\circ}=1$. Um quilobyte corresponde a $2^{\circ} \cdot 2^{10}=2^{10}$ bytes; um megabyte corresponde a $2^{10} \cdot 2^{10}=2^{20}$ quilobytes; um gigabyte equivale a $2^{20} \cdot 2^{10}=2^{30}$ megabytes.

Além das unidades de medida de quantidade de informação que você estudou no anterior, há outras. Veja:

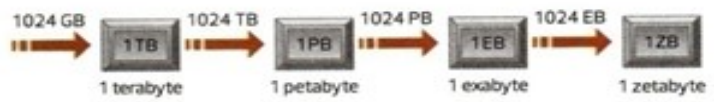

Após a leitura do texto, reallze as atividades propostas.

a) Copie a tabela abaixo e complete a sequência aplicando as propriedades da potenciação.

\begin{tabular}{|c|c|c|c|}
\hline byte & B & - & $2^{\circ}$ byte \\
\hline quilobyte & kB & $2^{\circ} \cdot 2^{\circ}$ & $2^{\circ}$ bytes \\
\hline megabyte & MB & $2^{10} \cdot 2^{10}$ & $2^{20}$ quillobytes \\
\hline gigabyte & GB & $2^{20} \cdot 2^{10}$ & $2^{10}$ megabytes \\
\hline terabyte & TB & $2^{30} \cdot 2^{10}$ & f \\
\hline petabyte & PB & falp & fotenongs: \\
\hline exabyte & EB & $z^{2}-2=$ & 2topente \\
\hline zetabyte & $z B$ & fantin & Aismoyns \\
\hline
\end{tabular}

b) Transforme 1,9 ZB em gigabytes (GB). $19 \cdot 1024^{*} \mathrm{~GB}$ ou $1.9 \cdot 2^{+0} \mathrm{~GB}$

c) Quantos pen drives de $8 \mathrm{~GB}$ são necessários para armazenar 92116 terabytes? $92116 \cdot 2$ ' ou 11790848 pen drives

Fonte: (DANTE, 2012b, p.113)

\section{Momento de exploração do tipo de tarefa $T_{i}$ e a sua articulação com alguma}

técnica $\tau_{i}$ : no volume 6 , as tarefas são apresentadas na seção: "Exercícios e problemas", enquanto que no volume 7 , as tarefas são apresentadas na seção: "Outros contextos". No volume 6 todas as tarefas referentes à quantidade de informação ou à velocidade de processamento de dados são apresentadas após a apresentação inicial de elementos 
tecnológico-teóricos. No volume 7, a técnica é articulada com as tarefas no próprio enunciado da questão 81 (Figura 3) na página 113. Chamamos esta técnica de técnica $\tau_{1}$. Ela busca converter unidades de medida de informação: para converter uma unidade de medida de informação em outra, basta multiplicá-la ou dividi-la por 1024. Ou seja, cada unidade de medida de informação é igual a 1024 vezes a unidade imediatamente inferior, tomando um procedimento semelhante ao deslocamento da vírgula para a direita ou para a esquerda. Nesses casos, são representados por meio de setas vermelhas. Enquanto à presença de técnica no volume 6, ela é implícita. No entanto, a conversão de unidades de medidas de mesma grandeza como, comprimento, área, capacidade, apresentadas neste mesmo volume, são dadas por meio do procedimento do deslocamento da vírgula para a direita ou para a esquerda.

Momento de constituição do bloco tecnológico-teórico: o livro do $6^{\circ}$ ano define as medidas de informação kilobyte, megabyte, gigabyte e terabyte em função de bytes e explica o significado de suas respectivas siglas (KB, MB, GB e TB) (Vide Figura 2). Ao lado, tem um desenho de um rapaz explicando por meio de um balão que "o correto seria: $1 \mathrm{~KB}=1024$ bytes; $1 \mathrm{MB}=1024 \mathrm{~KB} ; 1 \mathrm{~GB}=1024$ MB” (DANTE, 2012a, p.229). Justifica a aproximação de $1 \mathrm{~KB}$ igual a 1000B ao invés de $1 \mathrm{~KB}$ igual a 1024B para facilitar os cálculos. Segundo a literatura, $1 \mathrm{~KB}$ é igual a $1000 \mathrm{~B}$ e $1 \mathrm{KiB}$ é igual a $1024 \mathrm{~B}$, portanto, não existe aproximações de valores, mas sim representações de medidas diferentes: a primeira encontra-se no sistema decimal e a segunda encontra-se no sistema binário conforme exposto corretamente no volume 7: explica a diferença entre o Sistema Métrico Decimal e o Sistema de Medidas de Informação. O primeiro como sendo organizado por um sistema decimal (base 10) e o segundo como sendo organizado por um sistema binário (base 2).

\footnotetext{
No ano anterior $\left[6^{\circ}\right.$ ano], você viu que as unidades do Sistema Métrico Decimal de Medidas (litro, metro, grama, etc.) estão organizadas sobre a base 10. Ou seja, a partir de uma unidade padrão ou unidade fundamental, as demais unidades de medida são obtidas multiplicando-se ou dividindo-se por potências de base 10. Jà unidades como bit e bytes estão organizadas sobre a base 2, pois o bit pode assumir apenas dois valores, 0 ou 1 (sistema binário). Assim, um byte, entendido como unidade, corresponde a $2^{0}=1$. Um quilobyte corresponde a $2^{0} \cdot 2^{10}=2^{10}$ bytes; um megabyte corresponde a $2^{10} \cdot 2^{10}=2^{20}$ quilobytes; um gigabyte equivale a $2^{20} \cdot 2^{10}=2^{30}$ megabytes (DANTE, 2012b, p.113).
}

No entanto, apesar de explicar a diferença entre o sistema métrico decimal de medidas e o sistema de medidas de informação, os volumes 6 e 7 não adotam as nomenclaturas e nem os símbolos literais recomendados pelo SI: nomes e símbolos literais correspondentes aos 
números $2^{10}, 2^{20}, 2^{30}$ respectivamente a kibi, Ki; mébi, Mi e gibi Gi. Dessa maneira, os volumes 6 e 7 adotam o mesmo nome e o mesmo símbolo literal do sistema métrico decimal de medidas para representar as unidades de medida de informação, organizando-se desta maneira sobre a base 2 ao invés da base 10 .

Iremos adotar as nomenclaturas e símbolos literais do SI para explicar um equívoco na citação acima: um mébibyte corresponde a $2^{0} \cdot 2^{10}=2^{10}$ kibibytes ou a $2^{10} \cdot 2^{10}=2^{20}$ bytes e não a $2^{10} \cdot 2^{10}=2^{20}$ kibibytes, como presente na citação acima, assim como um gibibyte é igual a $2^{0} \cdot 2^{10}=2^{10}$ mébibytes ou $2^{10} \cdot 2^{10}=2^{20}$ kibibytes ou ainda, $2^{10} \cdot 2^{10} \cdot 2^{10}=2^{30}$ bytes e não a $2^{20} \cdot 2^{10}$ $=2^{30}$ megabytes, como presente na citação acima.

No volume 7, página 113, explica que a base 2 (sistema binário) utiliza-se apenas dois símbolos o 1 e o 0 para escrever um número binário. Define bit: "o bit é a menor unidade de informação que pode ser armazenada ou transmitida" (DANTE, 2012b, p.113). Define byte como sendo 8 bits. Comenta sobre a sua ampliação enquanto unidades de medida de informação: terabyte, petabyte, exabyte e zetabyte, e expõe seus valores. Estas definições não são apresentadas no livro do $6^{\circ}$ ano. O volume 6 , página 229 , representa as velocidades de processamento de dados, megahetz (MHZ) e gigahetz (GHZ), por ciclos por segundos:

$\mathrm{MHz}=$ megahertz $(1000000 \mathrm{~Hz}$ ou 1000000 ciclos por segundos $) \mathrm{e}$

$\mathrm{GHz}=$ gigahertz $(1000 \mathrm{MHz}$ ou $1000000000 \mathrm{~Hz}$ ou 1000000000 ciclos por segundos $)$

> Momentos de institucionalização: segundo o Manual do Professor, volume 7, páginas 4 e 5 , buscam integrar os 5 blocos de conteúdos da matemática básica, Geometria, Álgebra, Grandezas e Medidas, Estatistica e Probabilidade, e Números e Operações, de maneira que seja trabalhada de modo espiral ao longo dos 4 anos, retomando, ampliando e aprofundando conceitos e procedimentos já estudados. Além disso, busca-se trabalhar situações-problema contextualizadas para possibilitar a compreensão e atribuição de significados de tal maneira que evite a memorização e a mecanização. Em relação ao conteúdo estudado: medidas de informação e de processamento de dados, identificamos tarefas enquadradas como situações-problema contextualizado por meio de elementos da Informática. Enquanto a proposta de se abordar temas de maneira espiral, (neste caso, investigamos a abordagem de medidas de informação e de processamento de dados) constatamos a recapitulação, ampliação e aprofundamento do conteúdo de medidas de 
informação (do $6^{\circ}$ para o $7^{\circ}$ ano). O livro do $7^{\circ}$ ano define bit, byte (aprofundamento); distingui a organização de bases 2 e 10 entre as unidades de medida de informação e as unidades de medidas do sistema métrico decimal (aprofundamento); E amplia outras unidades de medida de informação: terabyte, petabyte, exabyte e o zetabyte (ampliação). O volume 7 informa ao leitor que está ampliando os conhecimentos dessa temática apresentado no livro do $6^{\circ}$ ano: "como você estudou no ano anterior, há diferentes grandezas e medidas relacionadas à informática, como por exemplo, as que envolvem armazenamento de dados” (DANTE, 2012, p.113).

Momento de trabalhar a técnica: o Manual do Professor do livro do $6^{\circ}$ ano unidade 2 - "Potenciação e divisibilidade" - capítulo 4 - "Potenciação, raiz quadrada e expressões numéricas", página 67, ilustra duas técnicas de conversão de um número representado no sistema decimal em um número representado no sistema de numeração binário sob o título: "Outras leituras" (Figura 5): método de divisões sucessivas por dois e método de representação por meio de uma expressão polinomial numérica de potências de base dois. No entanto, não justifica e nem explica qual é o significado dessas técnicas. $\mathrm{O}$ volume 7, na questão 81 , apresenta a conversão de unidades de medida de informação por meio de uma ideia semelhante à técnica de deslocamento de vírgula por meio de setinhas vermelhas (Figura 3). O que não deixa evidente qual é a operação a ser realizada (somar 1024 ? multiplicar 1024? elevar 1024 a potência?) para converter uma unidade de medida de informação em uma outra unidade superior. Portanto, as setinhas vermelhas em si e os valores de potências na correspondência entre unidades de medidas de informação e a sua unidade inferior na tabela da página 113 , volume 7 , são autoexplicativas às conversões entre unidades de medidas de informação.

Momento de avaliação: baseado no entendimento de que a avaliação é um conjunto de ações cujo objetivo, entre outros, é "diagnosticar como está se dando o processo ensinoaprendizagem e coletar informações para corrigir possíveis distorções observadas nele" (DANTE, 2012a, p. 29 - Manual do Professor) o volume 6 apresenta um único exercício referente às medidas de informação (Figura 4). Esta questão vem com uma foto de um computador, ao lado, com algumas configurações: memória RAM, disco rígido e memória de placa de vídeo. Solicita transformar as unidades de medida de informação gigabyte e megabyte em função de bytes. No livro do $7^{\circ}$ ano, após o texto sobre "armazenamento de 
dados na informática", página 113, solicita três questões $(\mathbf{a}, \mathbf{b}, \mathbf{c})$. A primeira pede para completar uma tabela onde estão presentes unidades byte e seus múltiplos, até zetabyte, aplicando as propriedades da potenciação que, por sinal, retoma o conhecimento de potenciação: "copie a tabela abaixo e complete a sequência aplicando as propriedades da potenciação" (DANTE, 2012a, p.113, item a).

Figura 4: Questão 15

15. A escola onde Carla estuda ganhou da comunidade do bairro um computador com as caracteristicas ao lado.

Escreva estas medidas, usando o byte como unidade e a potenciação:

a) Medida da capacidade de armazenamento do disco rigido. $\quad \begin{aligned} & 75 \times 10^{\circ} \mathrm{B} \text { (bytes) } \\ & (750 \mathrm{~GB}=750000\end{aligned}$

b) Medida damemória da placa de video. $256 \times 10^{\circ} \mathrm{B}$ (bytes) $(256 \mathrm{MB}=256000000 \mathrm{~B}$

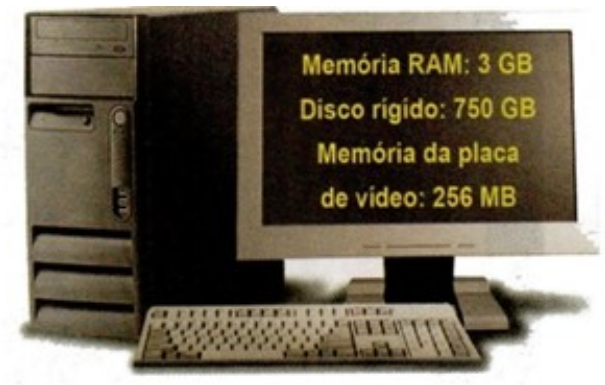

Fonte: (DANTE, 2012a, p.231, ex: 15)

Figura 5: Manual do Professor

\section{Sistema de numeração binária ou de base 2}

o número 123 e uma abreviaçăo de $1 \times 100+2 \times 10+3$ ou de $1 \times 10^{2}+2 \times 10+3 \times 10^{0}$. Dizemos entoa que 123 fol escrito na base 10 ou usando potencias de 10.

No lugar de potèncias de 10, vamos usar poténcias de $2(1,2,4,8,16,32 \mathrm{e} 64)$.

$123=1 \times 64+1 \times 32+1 \times 16+1 \times 8+0 \times 4+$ $+1 \times 2+1$

$123=1 \times 2^{4}+1 \times 2^{4}+1 \times 2^{4}+1 \times 2^{y}+0 \times 2^{2}+$ $+1 \times 2^{i}+1 \times 2^{\circ}$ ou, abreviando: $123=1111011_{\text {sane? }}$

Dizemos que 123 na base 10 pode ser escrito como 1111011 na base 2 ou como poténcias de 2 .

A maneira pratica de obter isso e fozer sucessivamente a divisäo por 2 :

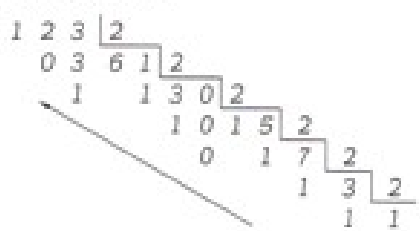

Lendo o ulitimo quodente e os restos de baixo para cima, temos:

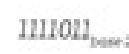

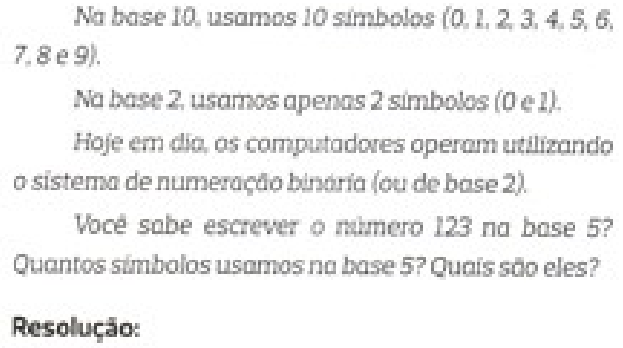

Na base 2, usamos apenas 2 simbolos ( 0 e 1 )

Hoje em dia, as computadores operam utilizando o sistema de numeraçao binaria (ou de base 2).

Voce sabe escrever o numero 123 na base 5? Quantos simbolos usamos na base 5 ? Quais sôo eles?

Resolução:

$$
123 \frac{5}{324 \frac{5}{4}}
$$

Respostas: $443_{\text {ane }}$; 5 simbolos: 0, 1, 2, 3e 4 .

Fonte: (DANTE, 2012a, p.67 - Manual do Professor) 


\section{Conclusão}

A abordagem dos números binários nos livros analisados (volumes 6 e 7) caracteriza-se como sendo uma praxeologia pontual e local. Ou seja, identificamos 1 tipo de tarefa $\left(\mathrm{T}_{61}\right)$ no volume 6 e 2 tipos de tarefas $\left(\mathrm{T}_{71}\right.$ e $\mathrm{T}_{72}$ no volume 8 .

$\mathrm{T}_{61}$ : determinar a quantidade de informação existente em um determinado artefato tecnológico;

$\mathrm{T}_{71}$ : transformar unidades de medida de informação e determinar a quantidade de artefatos tecnológicos (digitais) necessários para armazenar determinada medida de informação;

T72: corresponder corretamente às unidades de medida com a sua grandeza ou com a imagem de determinado objeto.

Identificamos uma técnica no volume 6 , referente à conversão de unidades de medida de mesma grandeza. Adaptamo-la, por organização sobre a base 2 .

$\tau_{1}$ : técnica do deslocamento de vírgula para a direita ou para a esquerda, realizando a multiplicação ou a divisão por 1024.

Identificamos duas técnicas ( $\tau_{2}$ e $\left.\tau_{3}\right)$ no Manual do Professor do volume 6 , referente à conversão de um número representado no sistema decimal em um número representado no sistema binário. No entanto, não é sugerida nenhuma tarefa referente a esse tipo de conversão.

$\tau_{2}:$ técnica das divisões sucessivas por 2

$\tau_{3}$ : técnica da representação por meio de uma expressão polinomial numérica de potências de base dois.

Identificamos uma tecnologia $\theta_{1}$, abordada discretamente no volume 6 por igualdades de unidades de medida de armazenamento de dados em função de bytes e de medida de velocidade de processamento de dados em função de ciclos por segundo. Sendo complementadas e aprofundadas no volume 7, por definir bit e byte, e apresentar outros múltiplos de bytes: terabyte, petabyte, exabyte e zetabyte. Não consideram as nomenclaturas e nem os símbolos literais sugeridos pelo SI (2014) e CIE (2005).

Não identificamos nenhuma teoria. 
Portanto, em um total de 2 tarefas ( 1 questão) no volume 6 e 2 tarefas ( 1 questão) no volume 7, identificamos 3 tipos de tarefas, 3 técnicas, uma única tecnologia e nenhuma teoria (Quadro 1 e Quadro 2).

Quadro 1: Praxeologia Pontual - volume 6

\begin{tabular}{|c|c|c|c|c|c|}
\hline Tipos de Tarefa & Página & Questão & Técnica & Tecnologia & Teoria \\
\hline $\mathrm{T}_{61}$ & 231 & $15 \mathrm{~b}$ e $15 \mathrm{c}$ & $\tau_{1}$ & $\theta_{1}$ & - \\
\hline
\end{tabular}

Quadro 2 - Praxeologia Local - volume 7

\begin{tabular}{|c|c|c|c|c|c|}
\hline Tipos de Tarefa & Páginas & Questões & Técnica & Tecnologia & Teoria \\
\hline $\mathrm{T}_{71}$ & 113 & $81 \mathrm{~b}$ & $\tau_{1}$ & $\theta_{1}$ & - \\
\hline $\mathrm{T}_{72}$ & 113 & $81 \mathrm{c}$ & & & \\
\hline
\end{tabular}

\section{Referências Bibliográficas}

BRASIL. Guia de Livros Didáticos PNLD 2014: Matemática: anos finais do ensino fundamental, Brasília: MEC, 2013.

CIE- Commission Electrotechnique Internationale Norme Internacionale - 60027-2: 2005, $3^{\mathrm{a}}$ edição, 2005.

CHEVALLARD, Yves. Analyse des pratiques enseignates et didactique des mathematiques: l'approche anthropologique, $1998 . \quad$ Disponível em $<$ http://yves.chevallard.free.fr/spip/spip/article.php3?id article=27\&var recherche=Analyse + des + pratiques + enseignates + et + didactique $+>$ Acesso em abril 2014.

CHEVALLARD, Yves. Les problématiques de la recherché en didactique à la lumiére de la TAD, 2011. Disponível em: $<$ http://yves.chevallard.free.fr/spip/spip/article.php3?id_article $=208 \&$ var_recherche $=$ Les + pro bl\%E9matiques + de + la + recherch $\% \mathrm{E} 9+\mathrm{en}+$ didactique $+\% \mathrm{E} 0+\mathrm{la}++>$ Acesso em abril 2014.

CHEVALLARD, Yves. Anthropolical approaches in mathematics educations, French perspectives. Encyclopedia of Matematics Education. Article: 313188, Chapter: 9, 2013.

DANTE, Luiz Roberto. Projeto Teláris: Matemática. $6^{\circ}$ ano. 1 ed. São Paulo: Ática, 2012. , Luiz Roberto. Projeto Teláris: Matemática. $7^{\circ}$ ano. 1 ed. São Paulo: Ática, 2012. , Luiz Roberto. Projeto Teláris: Matemática. $8^{\circ}$ ano. 1 ed. São Paulo: Ática, 2012. , Luiz Roberto. Projeto Teláris: Matemática. $9^{\circ}$ ano. 1 ed. São Paulo: Ática, 2012. 
MERTENSEN, Marianne. Praxeology as a tool for the analysis of a science museum exhibit. pp. 217-224. In Bosch, M. (Eds.) Um panorama de la TAD. Centre de Recerca Matemática, Campus de Bella-Terra Barcelona, 2011. ISSN: 2014-2323. Eletrônica: 2014-2331. III Congreso Internacional sobre la TAD (Sant Hilari Sacalm, 25-29 enero 2010).

SI: Le Système international d'Unités, 2014. Disponível em:

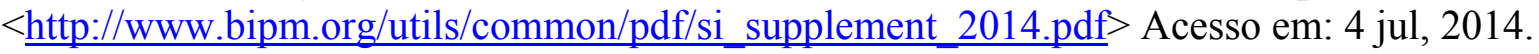

